\title{
Self-driven jamming in growing microbial populations
}

\author{
Morgan Delarue $^{1 \dagger}$, Jörn Hartung ${ }^{2 \dagger}$, Carl Schreck ${ }^{1}$, Pawel Gniewek ${ }^{1,3}$, Lucy Hu ${ }^{4}$, Stephan Herminghaus ${ }^{2}$ \\ and Oskar Hallatschek ${ }^{1,2 \star}$
}

In natural settings, microbes tend to grow in dense populations $^{1-4}$ where they need to push against their surroundings to accommodate space for new cells. The associated contact forces play a critical role in a variety of population-level processes, including biofilm formation ${ }^{5-7}$, the colonization of porous media ${ }^{8,9}$, and the invasion of biological tissues ${ }^{10-12}$. Although mechanical forces have been characterized at the single-cell level ${ }^{13-16}$, it remains elusive how collective pushing forces result from the combination of single-cell forces. Here, we reveal a collective mechanism of confinement, which we call self-driven jamming, that promotes the build-up of large mechanical pressures in microbial populations. Microfluidic experiments on budding yeast populations in space-limited environments show that self-driven jamming arises from the gradual formation and sudden collapse of force chains driven by microbial proliferation, extending the framework of driven granular matter ${ }^{17-20}$. The resulting contact pressures can become large enough to slow down cell growth, to delay the cell cycle in the G1 phase, and to strain or even destroy the micro-environment through crack propagation. Our results suggest that self-driven jamming and build-up of large mechanical pressures is a natural tendency of microbes growing in confined spaces, contributing to microbial pathogenesis and biofouling ${ }^{21-26}$.

The simultaneous measurement of the physiology and mechanics of microbes is enabled by a microfluidic bioreactor ${ }^{27-30}$ that we have designed to culture microbes under tightly controlled chemical and mechanical conditions. The set-up, shown in Fig. 1a, is optimized for budding yeast (Saccharomyces cerevisiae). We use this device to measure mechanical forces generated by partially confined growing populations and the impact of those forces on both the population itself and its micro-environment.

At the beginning of each experiment, we trap a single yeast cell in the growth chamber of the device, which can hold up to about 100 cells. The cells are fed by a continuous flow of culture medium, provided by a narrow set of channels that are impassable for cells.

Although cells first proliferate exponentially as in liquid culture, their growth dynamics are drastically altered once the chamber is filled. At high density, cells move in a stop-and-go manner and increasingly push against the chamber walls. The population develops a contact pressure that increases over time until it reaches a steady state, subject to large fluctuations. Note that this contact pressure is conceptually very different from the hydrostatic pressure, because water can flow in and out of cells. Depending on the geometry of the outlet (Fig. 1b,c), the mean steady-state pressure can reach up to $0.7 \pm 0.1 \mathrm{MPa}$. This pressure is larger than the osmotic pressure difference, approximately $0.2 \mathrm{MPa}$ (stationary phase $\mathrm{s}^{31}$ ), between the interior of a budding yeast cell and the surrounding medium, and much larger than the approximately $1 \mathrm{mPa}$ needed for the cells to overcome viscous friction (Supplementary Information).

Although the initial pressure build-up is similar in different devices, we find a sensitive dependence on the device geometry. The steady-state pressure can be finely tuned by the shape of the outlet gate (shown in Fig. 1b,c) or the width of the outlet channel (Supplementary Fig. 13).

Both the intermittent flow and pressure build-up are counterintuitive because, in all cases, the outlet channel is wide enough for cells to pass. In principle, excess cells could flow like a liquid out of the chamber. Time-lapse movies (Supplementary Movie 1) reveal that blockages in the device stabilize the cell packing and prevent flow. Cells proliferate until a sudden avalanche flushes them through the outlet (Fig. 1d,e). Another jamming event occurs, and the process repeats. These dynamics generate characteristic slow pressure increases followed by sudden pressure drops (Fig. 1c).

Jamming, intermittency and avalanches are familiar aspects of flowing sand, grains or even jelly beans ${ }^{24}$. To test whether the interplay of growth, collective rearrangement, and outflow of cells from the chamber can be explained by the mechanics of granular materials, we set up coarse-grained computer simulations with cells represented as elastic particles that grow exponentially and reproduce by budding. In our simulations, cells move by means of frictionless over-damped dynamics with repulsive contact interactions between neighbours.

Our simulations indeed reproduce the intermittent dynamics observed in the experiments (Fig. 2a-c). We find that the distributions of pressure drops have an exponential tail in both experiments and simulations (Fig. 2d) for $P>\langle P\rangle$, similar to avalanche size distributions in hopper flows ${ }^{32}$.

Highly intermittent cell flows might reflect spatially heterogeneous mechanical stresses, a hallmark of driven granular materials ${ }^{17-20}$. Assuming that cell shape deformation is indicative of the forces between cells, we developed a non-invasive method to infer these forces (Fig. 2f and Supplementary Information, and Supplementary Fig. 1). Using this approach, we analysed microscopy images to determine stress distributions of crowded populations. Both S. cerevisiae experiments and our coarse-grained simulations exhibit disordered cell packings that are stabilized by heterogeneous force networks (Fig. 2f,g). Stress is highly localized along branching 'force chains' ${ }^{\text {'17,18, }}$, whereas adjacent 'spectator cells $^{\prime 33}$ experience very little mechanical stress. 


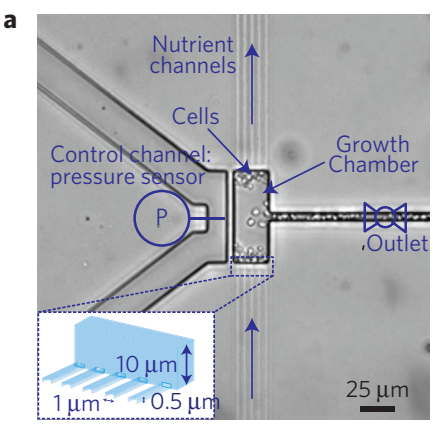

b
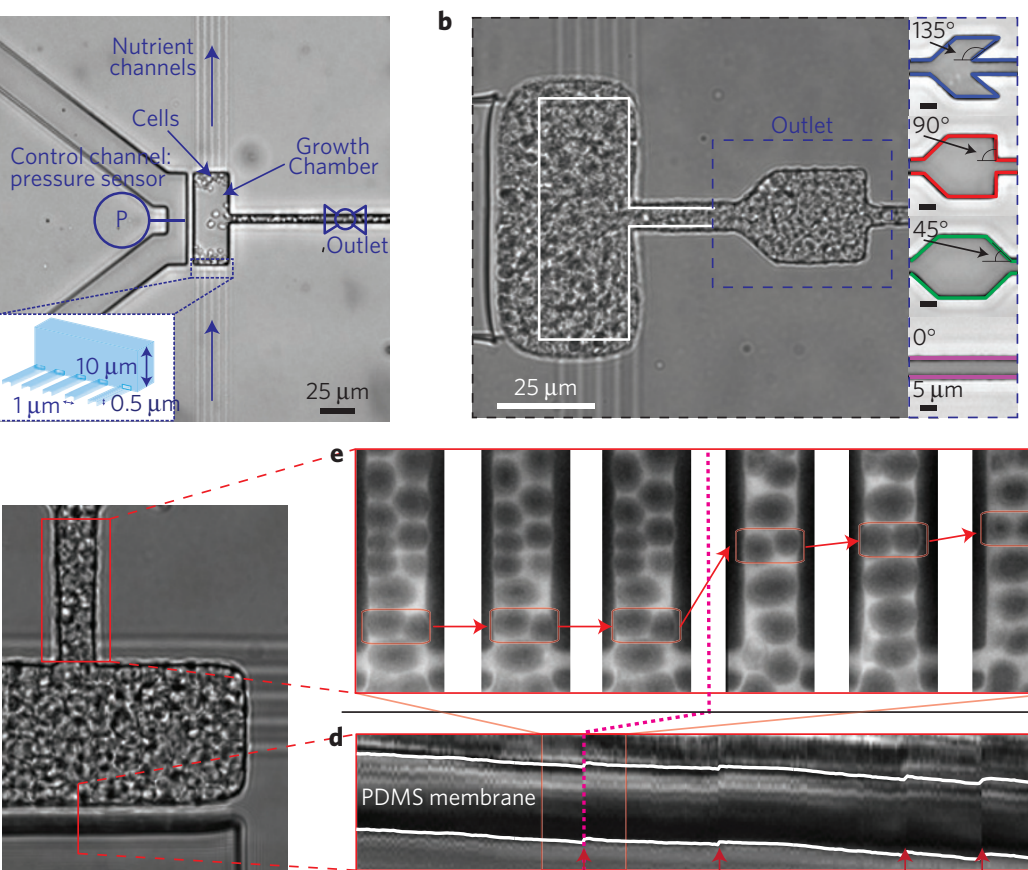
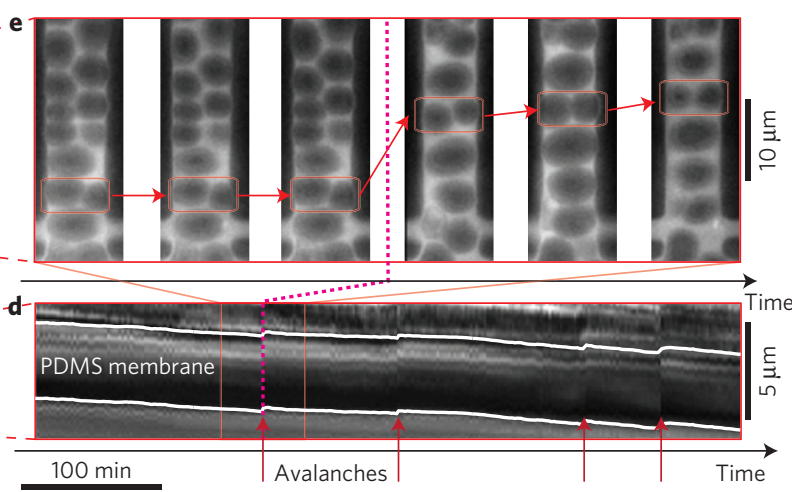

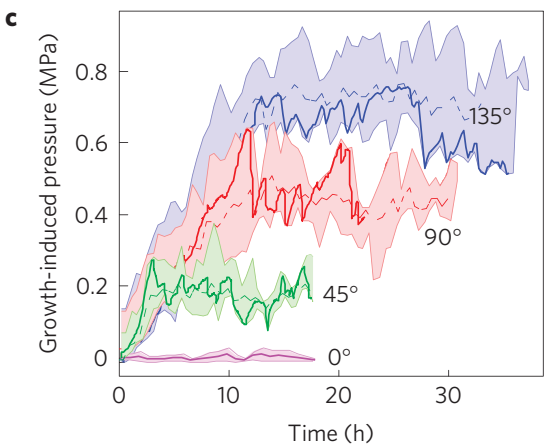

$\mathbf{f}$

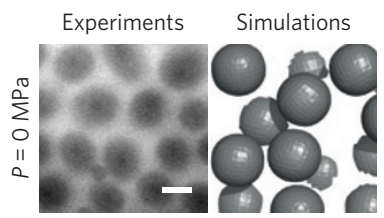

g

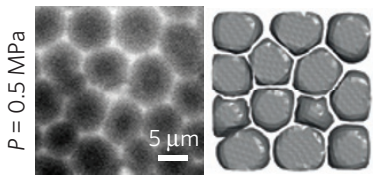

Figure 1 | Self-driven jamming of microbes enables collective pressure build-up in microfluidic environments. $\mathbf{a}$, Budding yeast cells are grown in a growth chamber threaded by narrow nutrient channels (inset). b. The jamming of excess microbes produced by proliferation in the device leads to a partial confinement of the population and a gradual build-up of a contact pressure of up to $0.7 \pm 0.1 \mathrm{MPa}$ (in the shown experiment), which strongly deforms the device (white line represents the undeformed layout). The steady-state pressure generated in a given device depends on the geometry of the outlets (b, right), which effectively act as leaky one-way valves. c, Resulting time-dependent pressure curves for the outlets shown in $\mathbf{d}$. The pressure measurements were enabled by an automatic feedback system that actively controls the deformation of a thin membrane separating the growth chamber and a control channel (see $\mathbf{a}$ and Supplementary Information). The bold curves correspond to one realization of the experiment, which is characterized by large pressure fluctuations due to gradual jamming and sudden unjamming. The shaded region represents the envelope of the replicates: all replicates are binned together and, within each bin, the minimum and the maximum define the shading. The dashed line corresponds to the mean of all realizations. The cellular flows exhibits collective features known from physics of jamming in granular media: the outflow of cells is not steady, but rather consists of periods of stasis, accompanied by pressure build-up, and sudden cell avalanches and pressure drops. This can be seen in time-lapse movies (Supplementary Movie 1) as well as kymographs. d,e, Random zig-zag motion of the chamber membrane (d) and flow through the outlet before, during and after an avalanche (e), with one snapshot every 20 min. $\mathbf{f}, \mathbf{g}$, Depending on the local stresses, cells assume shapes from nearly spherical (f, low stress) to nearly polyhedral ( $\mathbf{g}$, high stress). (Left) Micrographs taken close to the coverslip at the bottom of the chamber. (Right) Mass-spring simulations, in which cell walls are represented as (at vanishing contact pressure) spherical meshworks of springs (Supplementary Information). For better visualization, the simulations only show the first layer of cells. The depths of this layer are $5.25 \mu \mathrm{m}$ and $1.7 \mu \mathrm{m}$ for low and high pressure, respectively.

We find that jamming-induced contact forces can become so large that they feed back on the cell physiology. Indeed, a feedback on both cell shape and the dynamics of cell growth is evident in experiments where we place two devices of different steady-state pressures next to one another, as seen in the time-lapse movie (Supplementary Movie 2). These devices differ only in the width of their outlet channels ( $5 \mu \mathrm{m}$ versus $7.5 \mu \mathrm{m}$ ). We find that an increased outlet channel width leads to an increased mean avalanche size and, correspondingly, a smaller mean pressure (Supplementary Fig. 13). To quantify the feedback on growth, we estimate the net growth rate, which is the difference between birth and death rate, in our microfluidic bioreactors by measuring mean cell outflow rate at steady state (Supplementary Information). We find that the growth rate decays roughly exponentially with pressure until growth is undetectable at a stalling pressure of about $1 \mathrm{MPa}$ (Fig. 3c). The stalling pressure, or homeostatic pressure ${ }^{34}$, is obtained by using a special device with a 'self-closing valve', in which yeast populations fully confine themselves by the pressure they build up, as seen in Fig. 3a. In this device, the rate of pressure increase decays gradually with pressure until saturation (Fig. 3b). This diminishing return is due to smaller growth rates at higher pressures, and serves as another, dynamical measure for the feedback between contact pressure and growth rate.
Control experiments supported by finite-element simulations show that cells are well-fed and viable even at the highest densities, suggesting a mechanobiological origin for the reduced growth rates (Supplementary Information and Supplementary Figs 3 and 4).

As a first step to uncover the mechanistic basis for the forcegrowth feedback, we have explored the impact of contact forces on the pace of cell cycle progression. In budding yeasts, the late G1 checkpoint Start, homologue to the mammalian Restriction point, controls the irreversible cell commitment to division ${ }^{35}$. Passing of the checkpoint requires multiple phosphorylations of the repressor Whi5, on which Whi5 is exported out of the nucleus until the cell cycle is completed. As a consequence, Whi5 is localized in the nucleus in the G1 phase before Start, and cytosolic otherwise (Fig. 3d, top). Using a mutant that express fluorescently labelled Whi5 thus enabled us to probe the cellular commitment to cell division. We found that an increased contact pressure is accompanied by an increase in the fraction of cells with nuclear Whi5 signal (Fig. 3d), suggesting a force-induced slowdown of the cell cycle in G1. This finding is consistent with the view of the late G1 checkpoint as an integrator of numerous stresses, including osmotic, chemical and heat shock stresses $^{36-38}$. Force-induced cell cycle arrest has been observed in mammalian cells $^{39,40}$, but the associated mechanical stresses are 


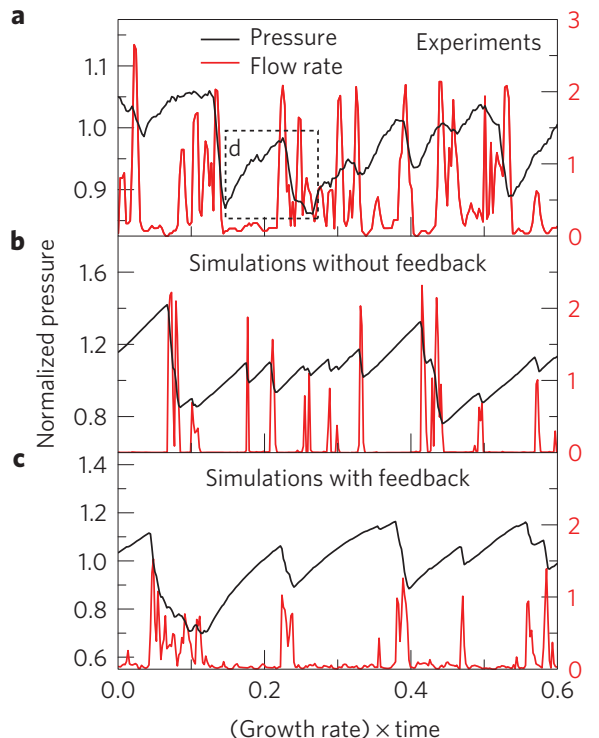

d
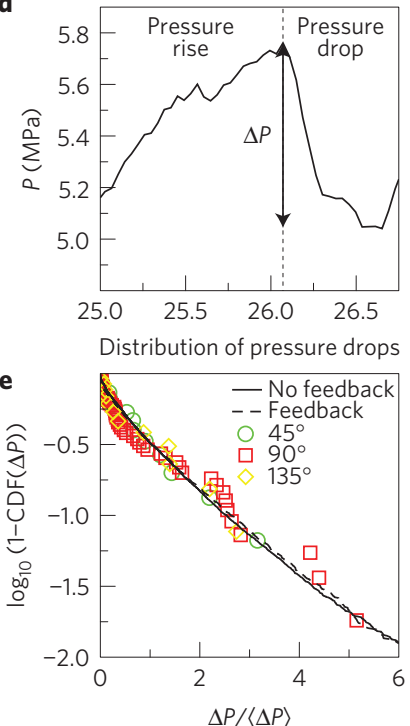

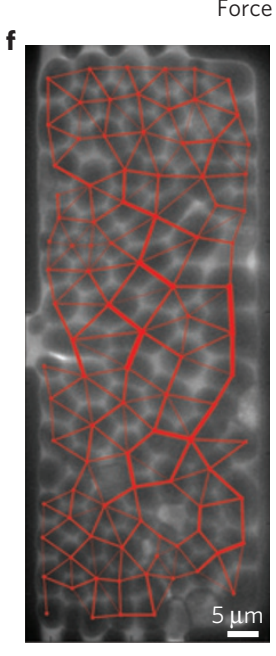

Experiments
Force networks

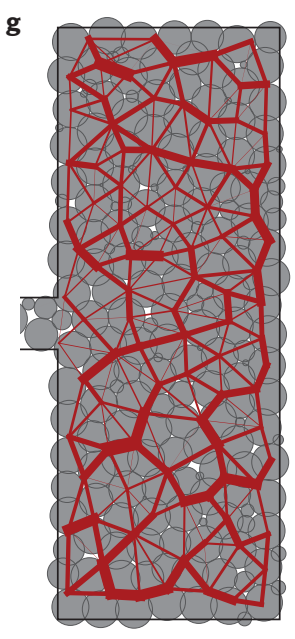

Simulations

Figure $\mathbf{2}$ | Pressure fluctuations and intermittent flows of partially confined budding yeast populations can be reproduced in simulations of proliferating elastic particles. a, Experimental pressure time series are characterized by periods of gradual pressure build-up and sudden pressure drops. $\mathbf{b}$, Simulations show that such time series are the generic outcome of jammed elastic particles proliferating in confined spaces. $\mathbf{c}$, A feedback of pressure onto growth, reported in Fig. 3c below, further improves our simulations. The gradual pressure increases before avalanche events show a diminishing return, similar to the experimental time series in $\mathbf{a}$. d,e, Pressure drops during avalanche events, defined as the pressure change from the peak pressure before an outflow event to the base pressure just after the event (d), are nearly exponentially distributed for drops larger than the mean pressure drop, $\langle\Delta P\rangle$, in both experiments (e: symbols) and coarse-grained simulations (e: lines). We can estimate inter-cell contact forces in our experiments by measuring the area of contact between two cells through image analysis. $\mathbf{f}$, The resulting network of contact forces in packings of budding yeast cells shows a heterogeneous distribution of mechanical stresses (pressure on the membrane: $0.5 \mathrm{MPa}$ ). $\mathbf{g}$, Force networks obtained from simulations of exponentially growing budding cells. In both $\mathbf{f}$ and $\mathbf{g}$, large forces are clustered into chain-like structures. Supplementary Movie 3 illustrates the dynamics of force networks in our experiments, and Supplementary Movie 4 is a coarse-grained simulation movie. For our simulations, we used box and outlet sizes that match the microfluidic chamber and parameterized the over-damped dynamics using the experimental flow rate and pressure fluctuation data (Supplementary Information).
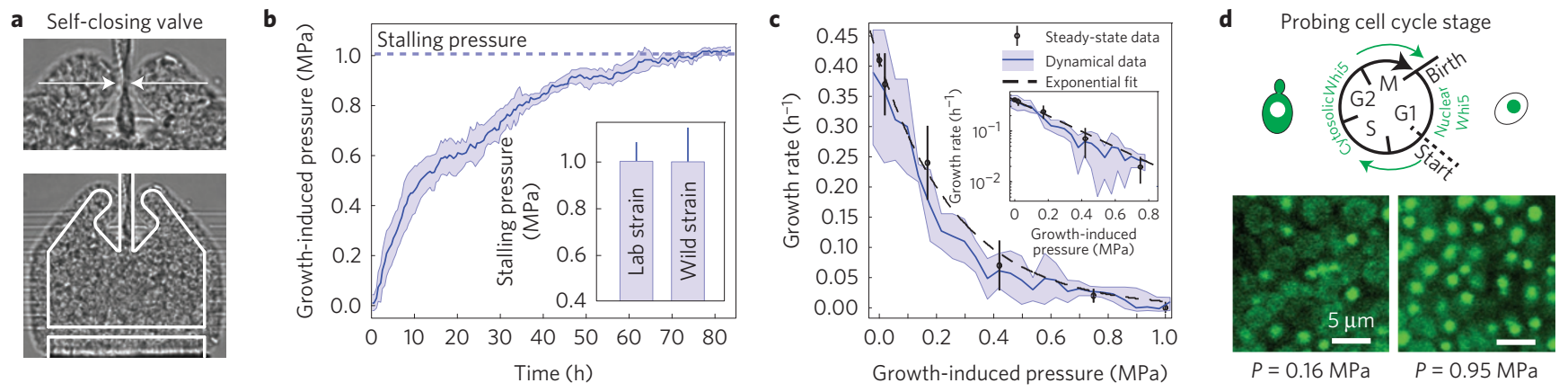

Figure $\mathbf{3}$ | Pressure-induced slow down of growth. a, Budding yeast populations can be fully confined using a 'self-closing' device that takes advantage of the contact pressure developed by the population to close the inlet/outlet channel. The cells are fed through narrow nutrient channels, as in 1a. The layout of the undeformed device is shown in white. $\mathbf{b}$, The time-dependent pressure curve in the self-closing devices shows a diminishing return: the rate of increase of the growth-induced pressure in the fully confined region gradually slows until it stops at the stalling pressure of $1 \pm 0.1 \mathrm{MPa}(5 \mathrm{replicates,} \mathrm{mean}$ \pm standard deviation). Inset: stalling pressure measured for the lab strain and the wild strain. c, Growth rate as a function of growth-induced pressure, estimated in two ways (Supplementary Information): the black points represent net growth rates determined from the cell flow out of our leaky devices in the steady state (black points; $\geq 5$ replicates, mean \pm standard deviation). The continuous blue line, on the other hand, has been inferred from the diminishing return in the dynamical data of $\mathbf{b}$ under a quasi-steady-state assumption (Supplementary Information; shading indicates \pm standard deviation). The dashed curves represents an exponential fit to the steady-state data $\left(k=0.41\left(h^{-1}\right) \exp (-P / 0.28(\mathrm{MPa}))\right)$. d, We probed the cell cycle progression using mutants that express fluorescently labelled Whi5 repressor proteins. In the G1 phase of the cell cycle before the checkpoint Start, Whi5 is localized in the nucleus, yielding a subcellular fluorescent focus (see scheme). We find that at high contact pressures of $0.95 \mathrm{MPa}$ almost four times as many cells exhibiting a nuclear Whi5 signal than at low pressures of $P=0.16 \mathrm{MPa}$ (Supplementary Fig. 5).

two to three orders lower than the stalling pressure measured in our experiments.

Perhaps the most salient consequence of growth-induced pressure is cell shape deformations. Whereas budding yeast cells grown in the absence of mechanical stresses are nearly spherical, we observe that they tend to morph into convex polyhedra as the population pressure becomes growth-limiting (Fig. 1f,g). Close to the stalling pressure, the packing resembles the structure of a dry foam $^{41}$, consisting of cells with nearly flat faces and sharp edges in between, shown in Fig. 2f. The pressure-induced cell shape 


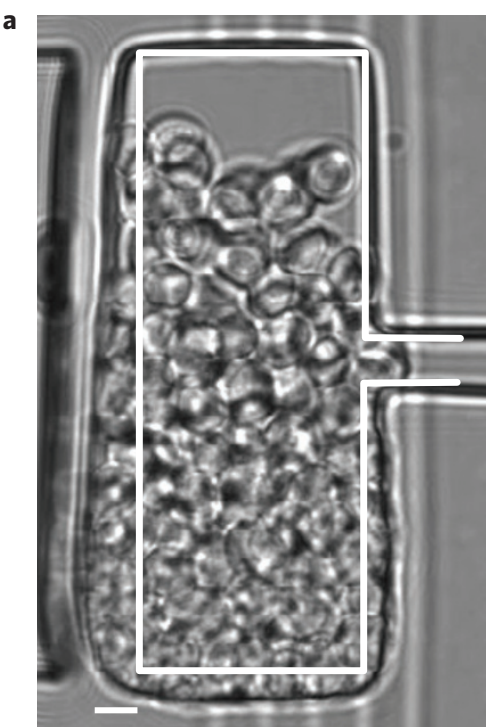

Wild strain

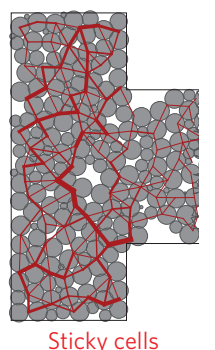

Sticky cells

c

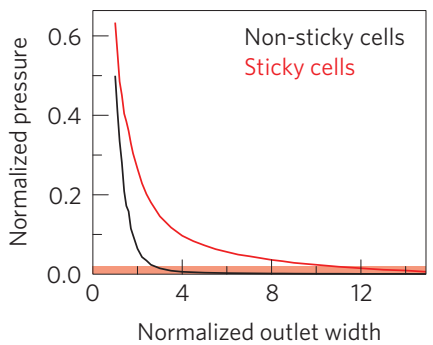

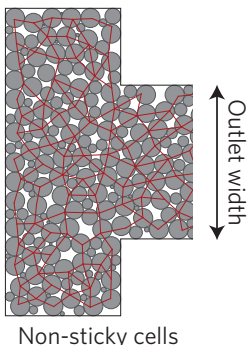

d
Local jamming propagates cracks in agar gel

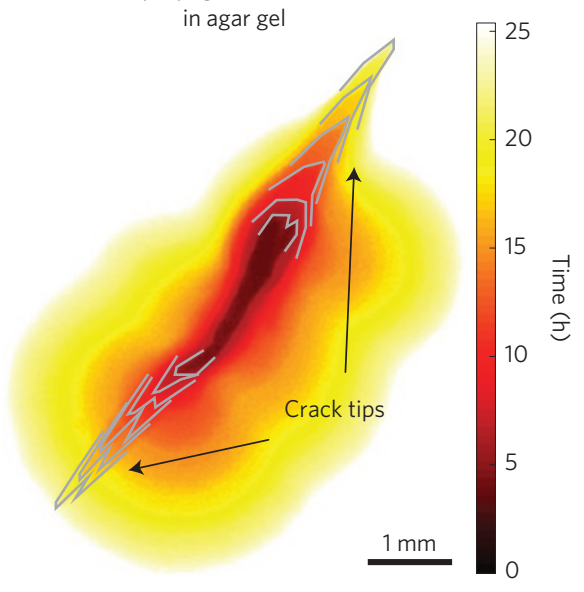

Figure 4 | Self-driven jamming is promoted by stickiness and can remodel the micro-environment. $\mathbf{a}$, Wild strains of yeast stick together via strong

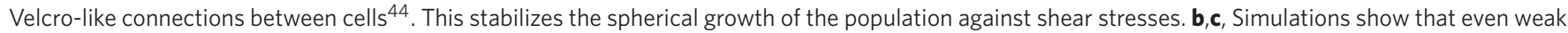

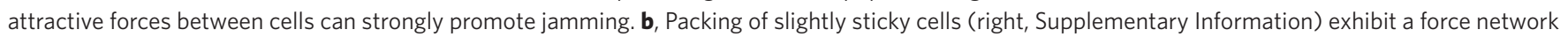

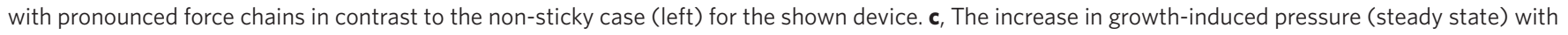

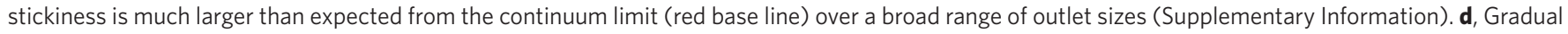
propagation of agar gel cracks by growing populations of budding yeast (lab strain). Cells grow out of a pre-existing agar crack and, at the same time, propagate the crack tips inside the agar. A time-lapse movie of the crack propagation is available (Supplementary Movie 5).

deformation can be best visualized at the interface between coverslip and cell population: the cell-coverslip contact area increases as the growth-induced pressure increases (Supplementary Fig. 6). Our simulations further suggest that, in our experiments, the osmotic pressure inside the cells may increase as a function of the growthinduced pressure (Supplementary Fig. 6).

Most microbial cells are sticky ${ }^{42,43}$. Indeed, whereas our lab strains of budding yeast have been domesticated to become non-sticky, wild strains can have strong, Velcro-like intercellular fibre connections ${ }^{44}$. We find that although sticky yeasts in our microfluidic devices develop a very similar maximal pressure as the lab strains do (Fig. 3b), they develop substantial contact pressures under much weaker confinement (Fig. 4a). Our coarse-grained simulations likewise suggest that attractive interactions promote jamming: the measured build-up of pressure is much larger than expected under a non-granular model of a liquid droplet with surface tension, in which jamming is impossible (Fig. 4c,d).

Bacteria and fungi have the ability to colonize a wide range of porous media, including tiny cavities barely larger than their cell size $\mathrm{e}^{3,4}$. Our work suggests that self-driven jamming of growing microbes can emerge in these micro-environments, as it does in our microfluidic devices, if chemical resources are sufficiently abundant.

The resulting growth-induced forces endow biofilms with the potential to remodel, or even destroy, their micro-environment. This could aid microbes in penetrating the soft tissues of host organisms ${ }^{10-12}$, or to invade soil, where most microbes grow in pores of several micrometres in diameter ${ }^{3,4}$. At this length scale, it is possible that the growth-induced pressures measured here contribute to straining of even stiff materials. Indeed, when we grow budding yeast populations inside agar gels, we observe the formation and propagation of cracks (Fig. 4d and Supplementary Fig. 8 and time-lapse movie Supplementary Movie 5). Thus, just like jamming of granular media can threaten the mechanical integrity of their confinements, which can lead to the bursting of grain silos ${ }^{32,45}$, it could also be an important mechanical aspect of host invasion ${ }^{10-12}$ and biofouling ${ }^{21}$.
We argue that the mechanism underlying self-driven jamming, cell proliferation, extends the notion of driven granular materials, which are usually jammed by external forces, such as shear, compression, or gravity ${ }^{17-20}$. On a fundamental level, cell proliferation and death are unique driving forces because they alter the number of macroscopic degrees of freedom, and thus directly affect Maxwellian rigidity criteria for jammed materials ${ }^{46,47}$. New granular physics may also result from biological features that have no analogue in traditionally driven granular materials. For instance, the pressure-growth feedback that we have described above could homogenize force networks and enhance pressure build-up, as our simulations indicate (Supplementary Fig. 11). Intermittent flows may be influenced by the shape of cells, as rod-like cells tend to align spontaneously, thus increasing the packing fraction ${ }^{48}$ (Supplementary Fig. 12). We also expect cell motility ${ }^{49}$ and viscoelastic extracellular substances ${ }^{6}$, expressed by many microbes to promote biofilm formation, to engage in a rich mechanical interplay with the packing of growing cells in confined spaces.

\section{Received 25 October 2015; accepted 23 March 2016; published online 9 May 2016}

\section{References}

1. Watnick, P. \& Kolter, R. Biofilm, city of microbes. J. Bacteriol. 182, 2675-2679 (2000).

2. Fanning, S. \& Mitchell, A. P. Fungal biofilms. PLoS Pathogens 8, e1002585 (2012).

3. Torsvik, V. \& Øvreås, L. Microbial diversity and function in soil: from genes to ecosystems. Curr. Opin. Microbiol. 5, 240-245 (2002).

4. Ranjard, L. \& Richaume, A. Quantitative and qualitatice microscale distribution of bacteria in soil. Res. Microbiol. 152, 707-716 (2001).

5. Wilking, J. N. et al. Liquid transport facilitated by channels in Bacillus subtilis biofilms. Proc. Natl Acad. Sci. USA 110, 848-852 (2013).

6. Wilking, J. N., Angelini, T. E., Seminara, A., Brenner, M. P. \& Weitz, D. A. Biofilms as complex fluids. MRS Bull. 36, 385-391 (2011).

7. Asally, M. et al. Localized cell death focuses mechanical forces during $3 \mathrm{~d}$ patterning in a biofilm. Proc. Natl Acad. Sci. USA 109, 18891-18896 (2012). 
8. Cunningham, A. B., Characklis, W. G., Abedeen, F. \& Crawford, D. Influence of biofilm accumulation on porous media hydrodynamics. Environ. Sci. Technol. 25, 1305-1311 (1991).

9. Rittman, B. E. The significance of biofilms in porous media. Wat. Resour. Res. 29, 2195-2202 (1993)

10. Gow, N. A. R., Brown, A. J. P. \& Odds, F. C. Fungal morphogenesis and host invasion. Curr. Opin. Microbiol. 5, 366-371 (2002).

11. Money, N. P. Turgor pressure and the mechanics of fungal penetration. Can. J. Bot. 73, 96-102 (2002).

12. Foster, T. J., Geoghegan, J. A., Ganesh, V. K. \& Höök, M. Adhesion, invasion and evasion: the many functions of the surface proteins of Staphylococcus aureus. Nature Rev. Microbiol. 12, 49-62 (2014).

13. Smith, A. E., Zhang, Z., Thomas, C. R., Moxham, K. E. \& Middelberg, A. P. J. The mechanical properties of Saccharomyces cerevisiae. Proc. Natl Acad. Sci. USA 97, 9871-9874 (2000).

14. Minc, N., Boudaoud, A. \& Chang, F. Mechanical forces of fission yeast growth. Curr. Biol. 19, 1096-1101 (2009).

15. Stenson, J. D., Thomas, C. R. \& Hartley, P. Modelling the mechanical properties of yeast cells. Chem. Eng. Sci. 64, 1892-1903 (2009).

16. Tuson, H. H. et al. Measuring the stiffness of bacterial cells from growth rates in hydrogel of tunable elasticity. Mol. Microbiol. 84, 874-891 (2012).

17. Radjai, F., Jean, M., Moreau, J. J. \& Roux, S. Force distribution in dense two-dimensional granular systems. Phys. Rev. Lett. 77, 274-277 (1996).

18. Majmudar, T. S. \& Behringer, R. P. Contact force measurement and stress-induced anisotropy in granular materials. Nature 435, 1079-1082 (2005)

19. Bi, D., Zhang, J., Chakraborty, B. \& Behringer, R. P. Jamming by shear. Nature 480, 335-358 (2011).

20. Heussinger, C. \& Barrat, J. L. Jamming transition as probed by quasistatic shear flow. Phys. Rev. Lett. 102, 218303 (2009).

21. Warscheid, T. H. \& Braams, J. Biodeterioration of stone: a review. Int. Biodeter. Biodegr. 46, 343-368 (2000)

22. Seebacher, C. et al. Onychomycosis. Mycosis 50, 321-327 (2007).

23. Douglas, L. J. Candida biofilms and their role in infection. Trends Microbiol. 11, 30-36 (2003).

24. GDR MiDi. On dense granular flows. Eur. Phys. J. E 14, 341-365 (2004).

25. Hall-Stoodley, L., Costerton, J. W. \& Stoodley, P. Bacterial biofilms: from the natural environment to infectious diseases. Nature Rev. Microbiol. 2, 95-108 (2004).

26. Park, J. A. et al. Unjamming and cell shape in the asthmatic airway epithelium. Nature Mater. 14, 1040-1048 (2015).

27. Rowat, A., Bird, J., Agresti, J., Rando, O. \& Weitz, D. Tracking lineages of single cells in lines using a microfluidic device. Proc. Natl Acad. Sci. USA 106, 18149-18154 (2009).

28. Cho, H. et al. Self-organization in high-density bacterial colonies: efficient crowd control. PLoS Biol. 5, e302 (2007).

29. Balagaddé, F. K., You, L., Hansen, C. L., Arnold, F. H. \& Quake, S. R. Long-term monitoring of bacteria undergoing programmed population control in a microchemostat. Science 309, 137-140 (2005).

30. Charvin, G., Cross, F. R. \& Siggia, E. D. A microfluidic device for temporally controlled gene expresion and long-term fluorescent imaging in unperturbed dividing yeast cells. PLoS ONE 3, e1468 (2008).

31. Martinez de Maranon, I., Maréchal, P. A. \& Gervais, P. Passive response of Saccharomyces cerevisiae to osmotic shift: cell volume variations depending on the physiological state. Biochem. Biophys. Res. Commun. 227, 519-523 (1996)

32. Zuriguel, I., Garcimartin, A., Maza, D., Pugnaloni, L. A. \& Pastor, J. M. Jamming during the discharge of granular matter from a silo. Phys. Rev. E 71, 051303 (2005).

33. Cates, M. E., Wittmer, J. P., Bouchaud, J. P. \& Claudin, P. Jamming, force chains, and fragile matter. Phys. Rev. Lett. 81, 1841-1844 (1998)

34. Basan, M., Risler, T., Joanny, J. F., Sastre-Garau, X. \& Prost, J. Homeostatic competition drives tumor growth and metastatic nucleation. HFSP J. 3 , 265-272 (2009).
35. Charvin, G., Oikonomou, C., Siggia, E. D. \& Cross, F. R. Origin of irreversibility of cell cycle start in budding yeast. PLoS Biol. 8, e1000284 (2010).

36. Escoté, X., Zapater, M., Clotet, J. \& Posas, F. Hog1 mediates cell-cycle arrest in G1 phase by dual targeting of Sic1. Nature Cell Biol. 6, 997-1002 (2004).

37. Shapiro, G. I. \& Harper, J. W. Anticancer drug tragets: cell cycle and checkpoint control. J. Clin. Invest. 104, 1645-1653 (1999).

38. Rowley, A., Johnston, G. C., Butler, B., Werner-Washburne, M. \& Singer, R. A. Heat shock-mediated cell cycle blockage and G1 cyclin expression in the yeast Saccharomyces cerevisiae. Mol. Cell. Biol. 13, 1034-1041 (1993).

39. Huang, S., Chen, C. S. \& Ingber, D. E. Control of cyclin D1, p27Kip1, and cell cycle progression in human capillary endothelial cells by cell shape and cytoskeletal tension. Mol. Biol. Cell 9, 3179-3193 (1998).

40. Delarue, M. et al. Compressive stress inhibits proliferation in tumor spheroids through a volume limitation. Biophys. J. 107, 1821-1828 (2014).

41. Weaire, D. \& Fortes, M. A. Stress and strain in liquid and solid foam. Adv. Phys. 43, 685-738 (1994).

42. Dufrêne, Y. F. Sticky microbes: forces in microbial cell adhesion. Trends Microbiol. 6, 376-382 (2015)

43. Soll, D. R. Candida biofilms: is adhesion sexy? Curr. Biol. 18, R717-R720 (2008)

44. Váchová, L. et al. Flo11p, drug efflux pumps, and the extracellular matrix cooperate to form biofilm yeast colonies. J. Cell Biol. 194, 679-687 (2011).

45. Dogangun, A., Karaca, Z., Durmus, A. \& Sezen, H. Cause of damage and failures in silo structures. J. Perform. Construct. Facil. 23, 65-71 (2009).

46. Maxwell, J. C. On the calculation of the equilibrium and stiffness of frames. Phil. Mag. 27, 294-299 (1864).

47. Wyart, M., Silbert, L. E., Nagel, S. R. \& Witten, T. A. Effect of compression on the vibrational modes of marginally jammed solids. Phys. Rev. E 72, 051306 (2005)

48. Volfson, D., Cookson, S., Hasty, J. \& Tsimring, L. S. Biomechanical ordering of dense cell populations. Proc. Natl Acad. Sci. USA 105, 15346-15351 (2008).

49. Bi, D., Yang, X., Marchetti, M. C. \& Manning, L. M. Motility-driven glass and jamming transitions in biological tissues. Preprint at http://arxiv.org/abs/1509.06578 (2015).

\section{Acknowledgements}

We would like to thank J. Rine, J. Thorner and L. Holt for helpful discussions, and $\mathrm{N}$. Podwitz and $\mathrm{H}$. Toncrova for their contributions at the initial stages of the project. Research reported in this publication was supported by the National Institute Of General Medical Sciences of the National Institutes of Health under Award Number R01GM115851, by a Simons Investigator award from the Simons Foundation (O.H.) and by the German Research Foundation (DFG) in the framework of the SFB 937/A15. The content is solely the responsibility of the authors and does not necessarily represent the official views of the National Institutes of Health.

\section{Author contributions}

O.H. designed and supervised the study. M.D., J.H., S.H. and O.H. designed the microfluidic experiments, M.D. and J.H. developed the software and performed experiments. M.D., J.H. and L.H. fabricated devices. M.D. and L.H. performed Comsol simulations, P.G. implemented and performed the mass-spring simulations, and C.S. implemented and performed the coarse-grained simulations. M.D., J.H., C.S., P.G. and O.H. interpreted the data and wrote the manuscript.

\section{Additional information}

Supplementary information is available in the online version of the paper. Reprints and permissions information is available online at www.nature.com/reprints. Correspondence and requests for materials should be addressed to O.H.

\section{Competing financial interests}

The authors declare no competing financial interests. 